be a critical factor in the development of ataxia. Without question, their data do suggest that reestablishing a normal DCN firing rate might have therapeutic potential.

1. Ramón y Cajal, S. 1888. Sobre las fibras nerviosas de la capa molecular del cerebelo. Rev. Trim. Histol. Normal Patol. 1:33-49.

2. Kim, J.J., and Thompson, R.F. 1997. Cerebellar circuits and synaptic mechanisms involved in classical eyeblink conditioning. Trends Neurosci. 20:177-181

3. Heslow, G., and Ivarsson, M. 1994. Suppression of cerebellar Purkinje cells during conditioned responses in ferrets. Neuroreport. 5:649-652.

4. Shakkottai, V.G., et al. 2004. Enhanced neuronal excitability in the absence of neurodegeneration induces cerebellar ataxia. J. Clin. Invest. 113:582-590. doi:10.1172/JCI200420216.

5. Aizenman, C.D., and Linden, D.J. 1999. Regulation of the rebound depolarization and spontaneous firing patterns of deep nuclear neurons in slices of rat cerebellum. J. Neurophysiol. 82:1697-1709.

6. Tomita, H., et al. 2003. Novel truncated isoform of SK3 potassium channel is a potent dominantnegative regulator of SK currents: implications in schizophrenia. Mol. Psychiatry. 8:524-535.

7. Cao, Y.-J., Drexler, J.C., Couey, J.J., and Houamed, K.M. 2002. Modulation of recombinant and native neuronal SK channels by the neuroprotective drug riluzole. Eur. J. Pharmacol. 449:47-54. 8. Lowe, J.S., and Leigh, N. 2002. Disorders of movement and system degenerations. In Greenfield's neuropathology. Volume 2. 7th edition. D.I. Graham and P.L. Lantos, editors. Arnold Publishing. New York, New York, USA/London, United Kingdom. 325-430.

9. Festoff, B.W., Suo, Z., and Citron, B.A. 2003 Prospects for pharmacotherapy of amyotrophic lateral sclerosis: old strategies and new paradigms for the third millennium. CNS Drugs. 17:699-717.

10. Raman, I.M., Gustafson, A.E., and Padgett, D. 2000. Ionic currents and spontaneous firing in neurons isolated from the cerebellar nuclei. J. Neurosci. 20:9004-9016

\section{The eosinophil enigma}

\author{
Timothy J. Williams
}

School of Medicine, Imperial College London, London, United Kingdom

Eosinophils accumulate in high numbers in the lungs of asthmatic patients. These cells have the ability to induce tissue damage, a capacity that relates to their traditional role in host defense against parasitic worms. On the other hand, eosinophils produce growth factors associated with tissue repair and remodeling, notably TGF- $\beta_{1}$. The relationship of these activities to lung dysfunction in asthma is highly controversial, but recent observations in humans and in animal models add spice to the debate (see the related article beginning on page 551).

J. Clin. Invest. 113:507-509 (2004). doi:10.1172/JCI200421073.

Since the discovery of the eosinophil, a leukocyte with a granular cytoplasm that stains pink with eosin dye, the function of this cell has been disputed; opinions on its role have swung periodically, as it has been characterized alternately as damaging and protective. Eosinophils accumulate in tissues in response to parasitic worm infections and, on activation, release cationic proteins from their granules and reactive oxygen species that are toxic to worms. Allergy may be an aberration of this defense process whereby otherwise innocuous agents in the environment stimulate an

\footnotetext{
Address correspondence to: Timothy J. Williams, Leukocyte Biology Section, Biomedical Sciences Division, School of Medicine, Imperial College London, Sir Alexander Fleming Building, Exhibition Road, London SW7 2AZ, United Kingdom. Phone: 44-020-7594-3159;

Fax: 44-020-7594-3119;

E-mail: tim.williams@imperial.ac.uk.

Conflict of interest: The author has declared that no conflict of interest exists.

Nonstandard abbreviations used:

bronchoalveolar lavage fluid (BALF).
}

adaptive immune response, characteristically driven by Th2 lymphocytes, resulting in the recruitment and activation of eosinophils that induce tissue damage. Eosinophils also release leukotrienes that induce smooth muscle contraction and microvascular leakage. IL-5, produced by Th2 lymphocytes, is critically important in this process, as it mediates the terminal differentiation and proliferation of eosinophils in the bone marrow and their release into the blood. IL-5 also primes eosinophils, for example, to facilitate eosinophil recruitment into tissues mediated by chemokines such as eotaxins, and IL-5 also prolongs eosinophil survival.

The key role of IL-5 in eosinophil production and function has made this cytokine a prime therapeutic target. This has been reinforced by results in animal models of asthma showing that deletion of the IL-5 gene (1) or the use of IL-5-neutralizing antibodies (2) is able to suppress eosinophil recruitment to the lung and, in many but not all cases (3), inhibit hyper-responsiveness of the airways to agents that induce bronchoconstriction, a characteristic feature of allergic asthma. Double eotaxin-1/IL-5 gene-deleted mice exhibited a greater suppression of eosinophil recruitment to the lung and the abolition of airway hyper-responsiveness associated, interestingly, with reduced IL-13 production (4).

Animal studies, particularly those in allergic cynomolgus monkeys showing long-term inhibition of lung eosinophilia and airway hyperresponsiveness by a single dose of an anti-IL-5 antibody (5), encouraged the initiation of clinical trials in which antibody was administered to mild atopic asthmatics (6) and chronic severe asthmatics (7). These studies showed no consistent effects on lung function tests, despite a marked suppression of blood eosinophils. From the results of these studies, some concluded that eosinophils are not causally related to lung dysfunction, although the results are contentious, particularly because of the small size of the trials and their technical limitations $(8,9)$. Furthermore, a followup study of bronchial biopsies from antibody-treated patients showed that despite marked depletion of blood and sputum eosinophils, airway tissue eosinophils were only depleted by $55 \%$ (10), suggesting that the residual cells could be important in lung dysfunction.

\section{The eosinophil in tissue remodeling}

The finding that eosinophils are able to produce growth factors directed attention to the potential role of these cells in the resolution of inflammation 


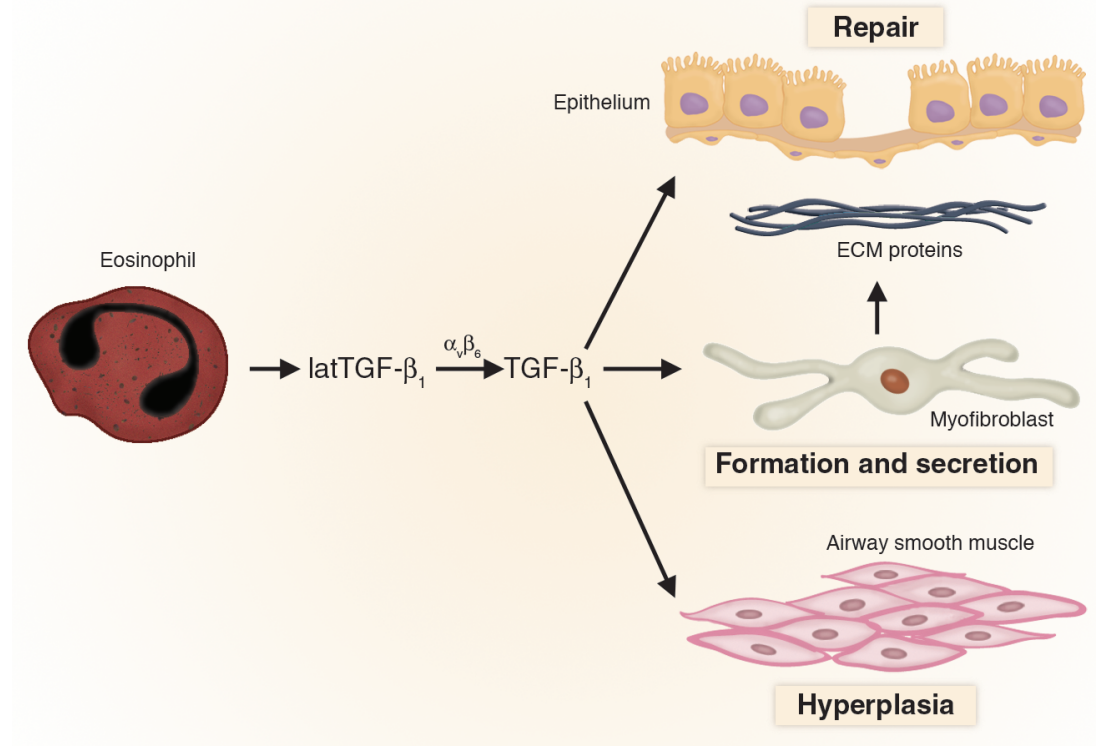

Figure 1

Role of eosinophil-derived TGF- $\beta_{1}$ in lung remodeling. Eosinophils in the asthmatic lung release latent TGF- $\beta_{1}$. Latent TGF- $\beta_{1}$ (latTGF- $\left.\beta_{1}\right)$ is activated by $\alpha_{v} \beta_{6}$ expressed on airway epithelial cells. TGF- $\beta_{1}$ mediates airway remodeling by inducing the formation of myofibroblasts that secrete extracellular matrix proteins, and the growth factor also stimulates airway smooth muscle hyperplasia.

and tissue repair. Tissue eosinophilia is a characteristic feature of several fibrotic diseases, and these cells are implicated in the remodeling that occurs in allergic asthma. Remodeling manifests itself in the airway wall as an increased deposition of extracellular matrix proteins in the reticular basement membrane and bronchial submucosa, an increase in smooth muscle mass, goblet cell hyperplasia, and angiogenesis. Eosinophils are capable of producing TGF- $\alpha$, TGF- $\beta_{1}$, and VEGF, as well as a range of cytokines, including IL-13.

Evidence from animal models supports the view that eosinophils have an important role in tissue remodeling. An antibody to IL-5 (11) and IL-5 gene deletion (12) have been shown to suppress lung eosinophilia and components of lung remodeling in murine models of allergic asthma. In this issue of the JCI, the study by Cho et al. (13) investigates the relationship between eosinophils and tissue remodeling in detail. They show in a chronic repetitive allergen challenge model that IL-5 gene deletion suppresses lung eosinophilia and tissue remodeling in parallel. Specifically, IL-5-deficient mice exhibited reduced eosinophil numbers in bronchoalveolar lavage fluid (BALF) and in peribronchial tissue. This was associated with decreased TGF- $\beta_{1}$ content of lung tissue with evidence that eosinophils were the major source of the growth factor. Interestingly, the epithelial cell expression of $\alpha_{v} \beta_{6}$, the integrin that is upregulated in response to tissue injury and activates latent TGF- $\beta_{1}(14,15)$, was also suppressed. Allergen-induced increase in peribronchial fibrosis, as measured by trichrome staining, collagen III and collagen $\mathrm{V}$ immunostaining, and total lung collagen content, was reduced in IL-5-deficient animals. Peribronchial smooth muscle thickness and epithelial mucus expression were also reduced.

These results echo observations made in humans. It has been shown that eosinophil-derived TGF- $\beta_{1}$ is associated with the transformation of fibroblasts to myofibroblasts and the expression by these cells of tenascin and procollagen I in allergic responses in human skin (16) (Figure 1). Furthermore, treatment of asthmatic patients with an antibody to IL-5 suppressed bronchial eosinophilia, the proportion of eosinophils expressing TGF- $\beta_{1}$, and the levels of TGF- $\beta_{1}$ in BALF (17). The expression of tenascin, lumican, and procollagen III in bron- chial mucosal reticular basement membrane was also reduced (17).

\section{The eosinophil as a therapeutic target}

All these observations suggest that the eosinophil, despite its checkered history, does make an important contribution to the pathology of allergic asthma. The effect of IL-5 neutralization or gene deletion on lung function varies between animal models (perhaps indicating different underlying mechanisms depending on experimental protocol), and such an effect using antibodies to IL-5 in humans has yet to be convincingly demonstrated. It may be that prolonged treatment targeted toward eosinophils may have an impact on symptoms as a consequence of suppression of alternating cycles of tissue damage and repair. If so, this will present a challenge in the design of appropriate clinical trials and prompt a reevaluation of biomarkers. An early study in guinea pigs showed that an antibody to IL-5 effectively suppressed allergen-induced BALF eosinophilia to low levels without affecting hyperresponsiveness, whereas higher antibody doses blocked hyper-responsiveness (18). This may relate to the recent clinical observations of residual eosinophils in lung tissue, or it may indicate actions of IL-5 that are independent of eosinophils. Either way, the doses of antibody used in published clinical trials may not have been high enough to demonstrate effects on lung function.

The studies discussed here may revive interest in other means of preventing eosinophil recruitment, particularly using chemokine receptor 3 antagonists. Combinations aimed at chemokines and IL-5 are attractive, but present considerable difficulties in testing and development.

Thus, the eosinophil appears to have the capacity to both injure and repair tissues, presumably related to its perceived traditional role in host defense. Knowledge of the underlying mechanisms may yet lead to therapy for allergic diseases, despite early setbacks.

\section{Acknowledgments}

T.J. Williams is the National Asthma Campaign Professor of Applied Pharmacology at Imperial College London. 
1. Foster, P.S., Hogan, S.P., Ramsay, A.J., Matthaei, K.I., and Young, I.G. 1996. Interleukin 5 deficiency abolishes eosinophilia, airways hyperreactivity, and lung damage in a mouse asthma model. J. Exp. Med. 183:195-201.

2. Hamelmann, E., et al. 1997. Antiinterleukin-5 antibody prevents airway hyperresponsiveness in a murine model of airway sensitization. Am. J. Respir. Crit. Care Med. 155:819-825.

3. Corry, D.B., et al. 1996. Interleukin 4 but not interleukin 5 or eosinophils, is required in a murine model of acute airway hyperresponsiveness. J. Exp. Med. 183:109-117.

4. Mattes, J., et al. 2002. Intrinsic defect in T cell production of interleukin (IL)-13 in the absence of both IL-5 and eotaxin precludes the development of eosinophilia and airways hyperreactivity in experimental asthma. J. Exp. Med. 195:1433-1444.

5. Mauser, P.J., et al. 1995. Effects of an antibody to IL-5 in a monkey model of asthma. Am. Rev. Respir. Dis. 152:467-472.

6. Leckie, M.J., et al. 2000. Effects of an interleukin-5 blocking monoclonal antibody on eosinophils, airway hyper-responsiveness, and the late asthmatic response. Lancet. 356:2144-2148.
7. Kips, J.C., et al. 2003. Effect of SCH55700, a humanized anti-human interleukin-5 antibody, in severe persistent asthma: a pilot study. Am.J. Respir. Crit. Care Med. 167:1655-1659.

8. O’Byrne, P.M., Inman, M.D., and Parameswaran, K. 2001. The trials and tribulations of IL-5, eosinophils, and allergic asthma. J. Allergy Clin. Immunol. 108:503-508.

9. Kay, A.B., and Menzies-Gow, A. 2003. Eosinophils and interleukin-5: the debate continues. Am.J Respir. Crit. Care Med. 167:1586-1587.

10. Flood-Page, P.T., Menzies-Gow, A.N., Kay, A.B., and Robinson, D.S. 2003. Eosinophil's role remains uncertain as anti-interleukin-5 only partially depletes numbers in asthmatic airway. Am. J. Respir. Crit. Care Med. 167:199-204.

11. Blyth, D.I., Wharton, T.F., Pedrick, M.S., Savage, T.J., and Sanjar, S. 2000. Airway subepithelial fibrosis in a murine model of atopic asthma: suppression by dexamethasone or anti-interleukin-5 antibody. Am. J. Respir. Cell Mol. Biol. 23:241-246.

12. Trifilieff, A., Fujitani, Y., Coyle, A.J., Kopf, M. and Bertrand, C. 2001. IL-5 deficiency abolishes aspects of airway remodelling in a murine model of lung inflammation. Clin. Exp. Allergy. 31:934-942.

13. Cho, J.Y., et al. 2004. Inhibition of airway remodeling in IL-5-deficient mice. J. Clin. Invest. 113:551-560. doi:10.1172/JCI200419133.

14. Huang, X.Z., et al. 1996. Inactivation of the integrin $\beta_{6}$ subunit gene reveals a role of epithelial integrins in regulating inflammation in the lung and skin. J. Cell Biol. 133:921-928.

15. Munger, J.S., et al. 1999. The integrin $\alpha_{v} \beta_{6}$ binds and activates latent TGF $\beta_{1}$ : a mechanism for regulating pulmonary inflammation and fibrosis. Cell. 96:319-328.

16. Phipps, S., et al. 2002. The relationship between allergen-induced tissue eosinophilia and markers of repair and remodeling in human atopic skin. J. Immunol. 169:4604-4612.

17. Flood-Page, P., et al. 2003. Anti-IL-5 treatment reduces deposition of ECM proteins in the bronchial subepithelial basement membrane of mild atopic asthmatics. J. Clin. Invest. 112:1029-1036. doi:10.1172/JCI200317974.

18. Mauser, P.J., et al. 1993. Inhibitory effect of the TRFK-5 anti-IL-5 antibody in a guinea pig model of asthma. Am. Rev. Respir. Dis. 148:1623-1627.

\section{HDL action on the vascular wall: is the answer NO?}

\section{Philip W. Shaul and Chieko Mineo \\ Department of Pediatrics, University of Texas Southwestern Medical Center, Dallas, Texas, USA}

Circulating levels of HDL cholesterol are inversely related to the risk of atherosclerosis, and therapeutic increases in HDL reduce the incidence of cardiovascular events. A new study (see the related article beginning on page 569) shows that HDL-associated lysophospholipids stimulate the production of the potent antiatherogenic signaling molecule NO by the vascular endothelium.

\section{J. Clin. Invest. 113:509-513 (2004). doi:10.1172/JCI200421072.}

The risk of cardiovascular disease from atherosclerosis is inversely proportional to serum levels of HDL and the major HDL apolipoprotein apoAI (1). In fact, low HDL levels predict an increased risk of coronary artery disease independently of LDL levels, and $60-70 \%$ of major cardiovascular

Address correspondence to: Philip W. Shaul, Department of Pediatrics, University of Texas Southwestern Medical Center at Dallas, 5323 Harry Hines Boulevard, Dallas, Texas 75390, USA. Phone: (214) 648-2015; Fax: (214) 648-2481;

E-mail: philip.shaul@utsouthwestern.edu. Conflict of interest: The authors have declared that no conflict of interest exists. Nonstandard abbreviations used: ATPbinding cassette transporter 1 (ABCA1); lysosulfatide (LSF); reverse cholesterol transport (RCT); scavenger receptor class B, member I (SR-BI); sphingosine-1-phosphate (S1P); sphingosylphosphorylcholine (SPC). events cannot be prevented with current approaches focused on LDL, such as statin therapy (2). In addition, low HDL levels are particularly common in males with early-onset atherosclerosis (3). Based on these observations, prevention trials have been performed with agents such as niacin and fibrates, which raise HDL, and they indicate that modest increases in HDL independently yield a significant reduction in cardiovascular events (4-6). Thus, there is compelling evidence that HDL is not solely a marker of lower risk of cardiovascular disease but instead is a mediator of vascular health.

Up until recently the protective features of HDL had been attributed primarily to its classical function of removing cholesterol from peripheral tissues and transferring it to the liver in a process known as reverse cholesterol transport (RCT). The delivery of cholesteryl ester from HDL to cells such as hepatocytes entails apoAI-mediated HDL cell surface interaction with scavenger receptor class $B$, member I (SR-BI), to which HDL binds with high affinity (7). Despite detailed understanding of HDL and RCT, the mechanisms by which HDL and apoAI are atheroprotective remain complex and not fully understood (8). This is particularly apparent when one considers evidence that circulating levels of HDL and apoAI do not regulate $\operatorname{RCT}(9,10)$, and that individuals with homozygous deficiency in the plasma cholesteryl ester transferase protein, which enhances HDL cholesterol delivery to the liver, have decreased risk of coronary artery disease (11).

Our basic understanding of the role of HDL in vascular biology has entered a new era in the past few years as direct modes of action of HDL on vascular cells have been elucidated. In particular, it has been demonstrated that HDL causes potent stimulation of eNOS activity through binding to SR-BI, which is expressed in endothelium (12, 13). Similarly, HDL enhances endothelium- and NO-dependent relaxation in aortas from wild-type but not SR-BIknockout mice (12). The HDL-induced increase in NO production may be critical to the atheroprotective features of HDL, as diminished bioavailablity of NO has a key role in the early pathogen- 